\title{
The burden of diarrhoeal disease in malnourished children
}

\section{Chandrika Murugaiah ${ }^{\mathrm{a}}$, Tin Swe Aye ${ }^{\mathrm{a}}$, Bagoes Soetjipto Soelyoadikoesoemo ${ }^{a}$, Harinarayan Radhakrishna ${ }^{a}$ and Lesley Maurice Bilung ${ }^{b}$}

\begin{abstract}
Diarrhoeal disease is the second leading cause of death in children, killing 1.5 million children every year. Malnourished children suffer most. Rotavirus, Escherichia coli, Shigella, Campylobacter, Salmonella, Vibrio cholerae and Cryptosporidium are the major pathogens that cause diarrhoeal disease in children. The tremendous incidence of diarrhoeal diseases in malnourished children underscores the urgent need for interventions, such as food-based oral rehydration salt (ORS) treatment. This review gives a summary of major pathogens that cause diarrhoeal diseases in poor nations and discusses the use of food-based ORS solutions in the treatment of dehydration in malnourished children. $\quad$ @ 2013 Wolters Kluwer Health | Lippincott Williams \& Wilkins
\end{abstract}

Reviews in Medical Microbiology 2013, 24:55-62

Keywords: dehydration, diarrhoea, emerging, infectious disease, microbiology

\section{Introduction}

Diarrhoea is caused by a wide range of pathogens, causing 1 billion disease episodes. Most episodes of childhood diarrhoea are mild, accounting for 840000 deaths among children under 5 years of age, according to the WHO. Diarrhoeal diseases certainly stand out clearly as a leading cause of childhood illnesses and death [1]. Diarrhoea is a disease of true poverty. More than half of these cases are reported in Africa and South Asia, an estimated 3-5 billion diarrhoeal illnesses occur annually in Africa, Asia and Latin America [2,3]. In some countries, diarrhoea is one of the most common causes of illness and death during infancy and early childhood [4-6]. It is the malnourished and poor [7], the poorest of the poor children in South Asian and sub-Saharan African countries, who are at high risk of getting the disease. In some countries, an average 2-12 episodes of diarrhoea per year per child has been reported [8-11]. Caused by various bacterial, viral and parasitic organisms, diarrhoea is defined as having loose or watery stools at least three times per day, or more frequently than normal for an individual. Among all, rotavirus infection is the leading cause of severe acute diarrhoea in young children $[12,13]$. Other major pathogens include Escherichia coli, Shigella, Campylobacter, Salmonella, Vibrio cholerae and Cryptosporidium $[2,12,14]$. They represent a major health problem in most countries, leading to death or other severe consequences if fluids are not replaced immediately. In poor countries, childhood mortality due to diarrhoea is not likely to decrease. The risk of dying is almost certainly greater among children suffering from malnutrition than among those suffering from diarrhoeal disease alone. Studies have shown that patients can be treated effectively with drinking an oral rehydration solution to combat dehydration $[15,16]$. The solution of salts and sugars developed in the 1960s is now saving over 1 million lives a year, including victims in rural areas and an epidemic setting $[15,17,18]$. This study gives a summary of major pathogens that cause diarrhoeal diseases in children. In addition, the present review is concerned with the implementation of recommendations for the treatment of the malnourished child, with the objectives of providing better nutrient, reducing/shortening illness and

\footnotetext{
${ }^{\mathrm{a} N e w c a s t l e}$ University Medicine Malaysia (NUMed), Kota Ilmu, EduCity@Iskandar, Nusajaya, Johor, and ${ }^{\mathrm{b}}$ Faculty of Resource Science and Technology, Universiti Malaysia Sarawak, Kota Samarahan, Sarawak, Malaysia.

Correspondence to Chandrika Murugaiah, Newcastle University Medicine Malaysia (NUMed), No.1, Jalan Sarjana 1, Kota Ilmu, EduCity@Iskandar, 79200 Nusajaya, Johor, Malaysia.
}

Tel: +60 19 9115040; fax: +60 9 7181700; e-mail: chandri20@yahoo.co.uk

Received: 4 February 2013; accepted: 19 March 2013. 